\title{
Energy Consumption Control of an Air Conditioner Using Web Services
}

\author{
Asaad Elmoudi, Omar Asad, Melike Erol-Kantarci, Hussein Talaat Mouftah \\ School of Information Technology and Engineering, University of Ottawa, Ottawa, Canada. \\ Email: \{aelmoudi, melike.erolkantarci, omar.asad, mouftah\}@uottawa.ca \\ Received February 18, 2011; revised March 18, 2011; accepted March 25, 2011.
}

\begin{abstract}
Air-conditioning (AC) systems have the highest power consumption among the appliances and consumer devices used at residential homes and buildings. Reducing their energy use will lower peak time usage and lower $\mathrm{CO}_{2}$ emissions. Recently, employment of the Information and Communications Technologies (ICT) to the power grid has smartened the grid. In the smart grid new opportunities emerge for AC energy consumption control. The aim of this paper is to reduce the air conditioning energy consumption of residential customers. It proposes an architecture that provides easy management and control using sensor network web services. A simulated thermal model of a house considering house data and outside temperature is presented. Simulation results showed a proposed temperature control method can have significant energy saving while maintaining customer comfort.
\end{abstract}

Keywords: Smart Grid, Web Services, Energy Management Systems

\section{Introduction}

Heating and cooling systems can account for up to $50 \%$ of the total energy consumed in buildings. Since the ACs consume significant amount of energy, it is reasonable to study the control of an $\mathrm{AC}$ with a focus on reducing its energy consumption. Reduced energy consumption has several immediate benefits. From consumers' point of view, their energy expenses and carbon footprints will be lower. From utilities' point of view, load will be reduced. Reducing the load is significant especially during peak hours since, as the load increases, the loss on the transmission lines increase. Furthermore, for extremely high loads, transformer overloading may occur and this may result in partial or full grid failures.

Due to the mentioned challenges, controlling the energy consumption of power equipments such as $\mathrm{AC}$ is significant. The energy consumption of an $\mathrm{AC}$ varies depending on the outside temperature. For cooling on a hot day (AC) or heating in a cold day (heater), the energy consumption can be very high. Those heating and cooling demands during extreme weather conditions may cause critical peaks and negatively impact the electrical network. To address this situation home energy management and web services techniques can be combined to control the consumption of the ACs.

In the smart grid, the capabilities of the demand re- sponse applications extend towards residential users where fine resolution demand control becomes possible. Energy management systems in the customer sector aims to reduce the electrical load of appliances, as well [1]. For instance, energy star appliances and energy-efficient light bulbs provide savings for energy consumption. However, by employing communications and control techniques further savings become possible. In [2], the authors presented an energy management and control system architecture for heating system in a house to reduce peak consumption while maintaining thermal comfort. A control algorithm based on measured power and inside temperature is proposed and implemented. In [3-4], an adaptive controller is implemented to control the AC consumption. Controllers can use energy estimation models that capture thermodynamics, power usage and thermostat control instead of direct measurements as proposed in this paper.

In fact, modeling the heating and cooling demand is a complex task where parameters such as outdoor temperature, house geometry, level and type of insulation, and type of equipment impact the model. There are various examples of modeling and simulating the energy consumption related with heating and cooling of a house [5-7].

Controlling the energy consumption of the ACs using Zigbee has been proposed in [2]. We use web services 
for their flexibility and suitability for machine to machine communications. There are two protocols that can be used in the web services, namely, SOAP and RESTfull. In [8], the authors have compared the performance of these protocols. The efficiency and the power consumption of RESTfull have been shown to be more efficient than SOAP. However SOAP is still widely used in web services as it is compatible with other applications. For this reason, in this paper we also use SOAP.

Web services are useful tools for machine to machine communications since they are platform independent. Recently, sensor web services have been gaining increasing interest from the research community. Using web services on the sensor nodes have several challenges related with memory and battery power limitations of the sensor nodes. In [9], the authors address these challenges by decreasing the TCP/IP overhead and customizing the XML messages on the node side via compression. In our implementation, we use a similar technique to decrease the XML and code sizes in the sensor nodes.

Sensor web services have been employed in IrisNet [10] which is a software platform allowing the users access the globally distributed sensors. Furthermore, using sensor web services in the smart grid applications have been initially proposed in [11]. The authors use sensor web service applications to enable energy savings and to improve the utilization of the renewable energy resources in the smart grid.

In this paper, an AC energy management and control architecture using web services is proposed. A thermal model of a house considering the outside temperature and house data is presented. Inside temperature control to reduce the energy consumption while maintaining the thermal comfort is proposed. Simulation results show that controlling the temperature can reduce the energy consumption significantly.

The rest of the paper is organized as follows. Section 2 describes the web services for AC energy management and control system and discusses the scalability of the application. In Section 3 the thermal model of a house is described. The energy consumption control is discussed in Section 4 and conclusion is provided in Section 5.

\section{AC Energy Management and Control using Web Services}

Generally energy management systems consist of several components which are sensors to measure and control variables; a controller able to carry out logical control operations or to provide control signals; actuator devices which receive the control signals and perform the required actions. Controllers are typically computing elements which implement load management via control programs. Controllers can use energy estimation models instead of direct measurements [3].

Figure 1 gives the web services implementation for the AC control in the smart grid.

In our implementation, the smart home containing the $\mathrm{AC}$ houses a sensor network and a sensor gateway node. The gateway node is between the sensor nodes on the AC and the internet. Web server makes remote function calls that invoke methods on the web service components in the smart home. The reply is sent in a compressed XML format to the server. The web service protocols run over the internet protocols. The gateway node can retrieve the information requested by the web server from the AC sensor by accessing its web service. Moreover, the utility and the inhabitants of the house can access the web server.

We implement the web services in Java NetBeans 6.8 IDE using the SOAP protocol and we use MySql server 5.1 to store the obtained data. We first analyze the scalability of the proposed $\mathrm{AC}$ control mechanism based on the completion time metric. The completion time of the application is the time needed for a single process to be completed. Once the web server generates a request (GetACReq), the timer registers the current timestamp as $T=$ $t_{0}$, then, the sensor receives the request packet and perform the required processing through the web service located on self. When the response packet (GetACReq) is ready on the sensor side, it is sent back to the web server through the internet. The time stamp $T=t_{1}$ is taken when the response reaches the server. The completion time is the difference between $t_{0}$ and $t_{1}$.

In Figure 2, we present the performance of the sensor web services in terms of completion time. We have increased the number of houses from three to 300 . For a typical distribution system, the completion time linearly increases with the number of controlled houses. For 300 houses the completion time reaches almost 3.7 seconds which is acceptable.

\section{Thermal Model of a House}

In this paper, our focus is on the cooling which can be easily extended to heating. We first describe the thermal model of the house. The cooling system is basically a heat energy removed from interior of a building in order to maintain desired comfort conditions. The thermal model equation is built based on the heat exchanged between the building and the environment is given by [7]:

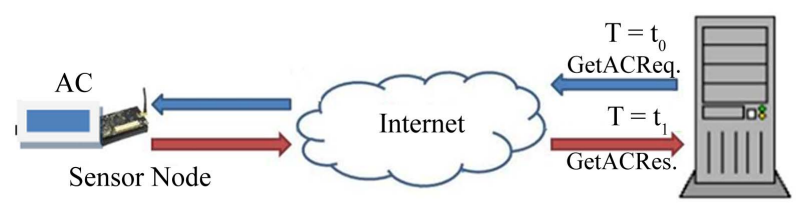

Figure 1. AC load control by web services. 


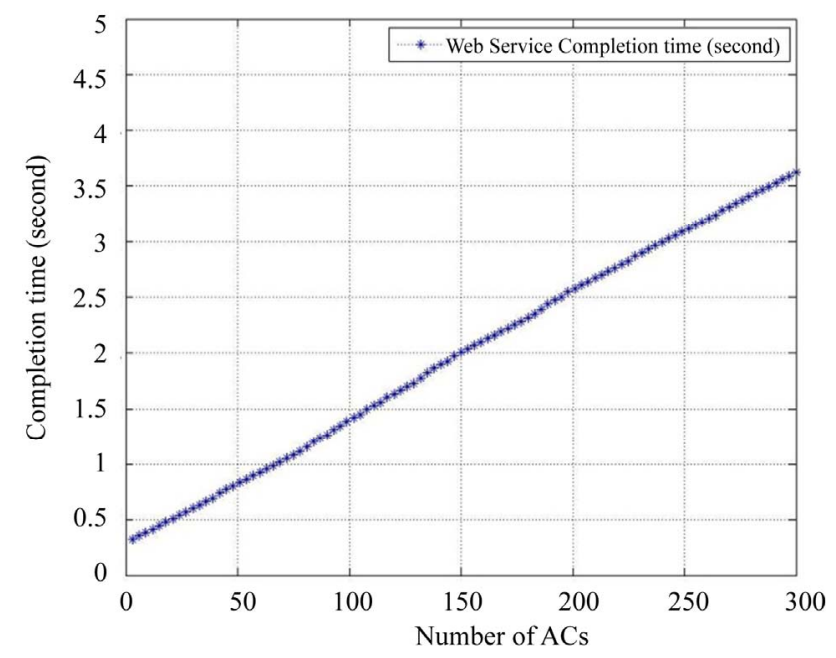

Figure 2. Performance of sensor web services.

$$
C \frac{\mathrm{d} \theta_{\text {in }}}{\mathrm{d} t}=-P(t)+\frac{1}{R}\left[\theta_{\text {in }}-\theta_{\text {out }}\right]
$$

where,

$\mathrm{P}(\mathrm{t})$ is the cooling power $\mathrm{W}$

$\Theta_{\text {in }}$ is the indoor temperature ${ }^{\circ} \mathrm{C}$

$\mathrm{C}$ is the heat capacity of indoor air $\mathrm{J} /{ }^{\circ} \mathrm{C}$

$\Theta_{\text {out }}$ is the outdoor temperature ${ }^{\circ} \mathrm{C}$

$\mathrm{R}$ is the equivalent thermal resistance of the building ${ }^{\circ} \mathrm{C} / \mathrm{W}$

The lumped thermal resistance of the house is calculated using the house dimension, number and size of windows and type of insulation used. Generally modern air conditioner is equipped with a thermostat. This thermostat is used to maintain the temperature at a desired temperature $\Theta_{\text {des. }}$. It trips the AC when the inside temperature goes below a minimal temperature and switches on the $\mathrm{AC}$ when inside temperature exceeds a maximal temperature. Figure 3 shows the simplified diagram of the thermal model. The model equation is implemented in MATLAB/Simulink and solved using Rung Kutta numerical method.

In the following set of results, the simulation model is applied to a house with central Air conditioning. Figure 4 shows the outside temperature of a hot summer day. Figures 5 and $\mathbf{6}$ show the simulated indoor temperature, the desired temperature $\Theta_{\mathrm{des}}$ i.e. the reference temperature and the $\mathrm{AC}$ operation for 24 hours for different temperature settings $20 \pm 1{ }^{\circ} \mathrm{C}$ and $23 \pm 1{ }^{\circ} \mathrm{C}$.

It can be seen that at high outside temperature and lower desired temperature the AC consumption increase as the AC takes longer on time to cool the house to the desired temperature. The calculated energy consumption for the $20 \pm 1{ }^{\circ} \mathrm{C}$ setting is calculated as shown in Figure 7. The energy consumed by the AC over 24 hours represents large consumption of any residential building; therefore controlling their consumption can bring benefits to customers and utilities.

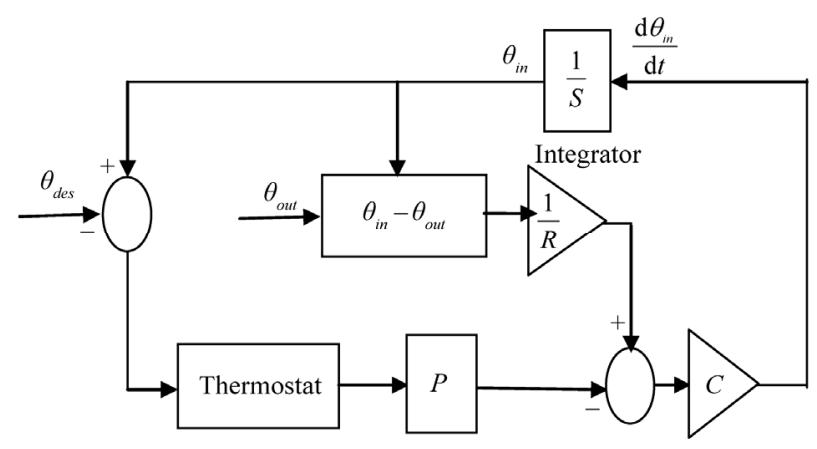

Figure 3. Simplified diagram of the thermal model.

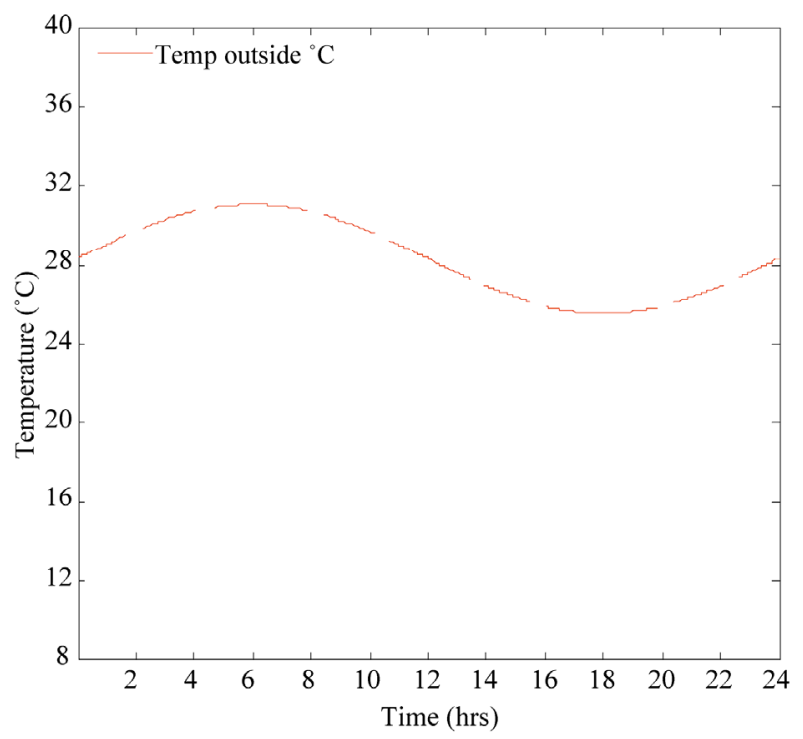

Figure 4. Outside temperature.

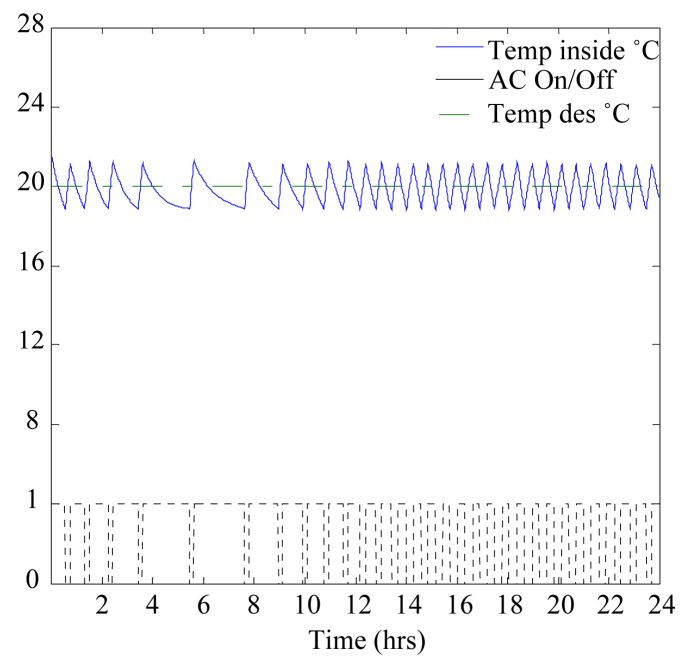

Figure 5. AC operation for $20^{\circ} \mathrm{C}$ desired temperature. 


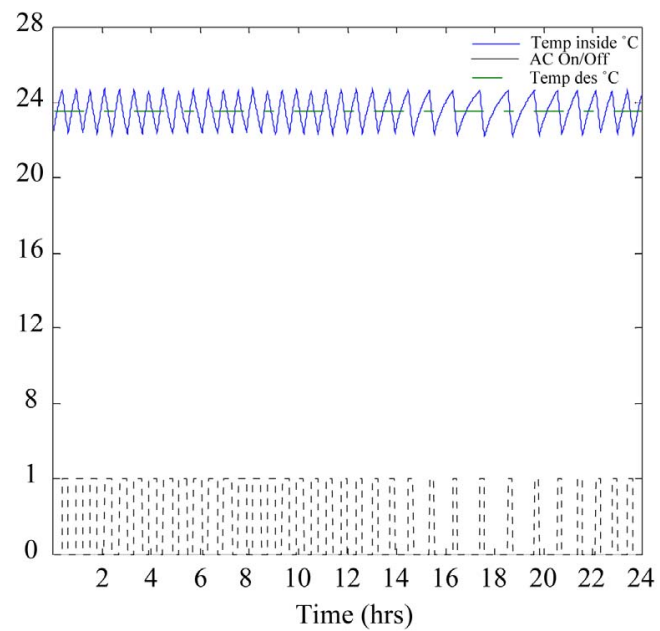

Figure 6. AC operation for $23^{\circ} \mathrm{C}$ desired temp.

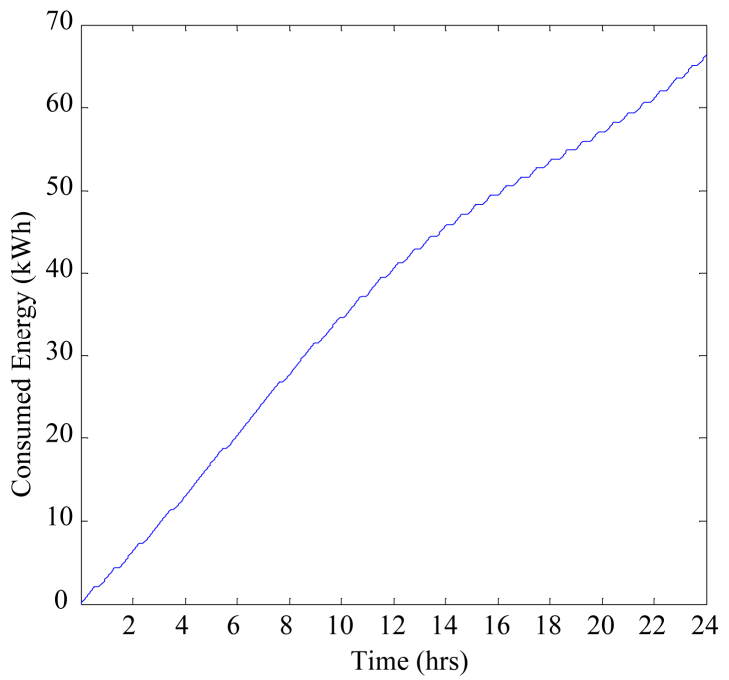

Figure 7. Energy consumption for $20^{\circ} \mathrm{C}$ desired temp.

\section{AC Energy Consumption Control}

The consumption can be controlled by changing the temperature inside the house within the thermal comfort. AC's are fitted with a simple hysteresis control i.e. thermostat to stay within a normal performance range. It is possible to modify load cycles without significantly impacting user comfort or convenience (e.g., inside temperature), thereby remaining undetectable to customers.

The thermal comfort depends on many factors such as temperature, humidity etc. However in this paper the convenience is based on the general recommendation by the Canadian center for occupational health and safety that the temperature to be held in the range of $21^{\circ} \mathrm{C}$ $23^{\circ} \mathrm{C}\left(69^{\circ} \mathrm{F}-73^{\circ} \mathrm{F}\right)[12]$. It is also advisable to keep the place slightly warmer to minimize the temperature discrepancy between indoor and outdoor [12].
We propose to set the minimum temperature and by varying the maximum temperature within the thermal comfort to achieve the required energy consumption reduction. The concept is clearly illustrated in Figures $\mathbf{8}$ and 9.

If the temperature $\Theta_{\min }$ is set to $20^{\circ} \mathrm{C}$ varying the temperature $\Theta_{\max }$ to a higher temperature, results significant energy consumption while the thermal comfort is main-

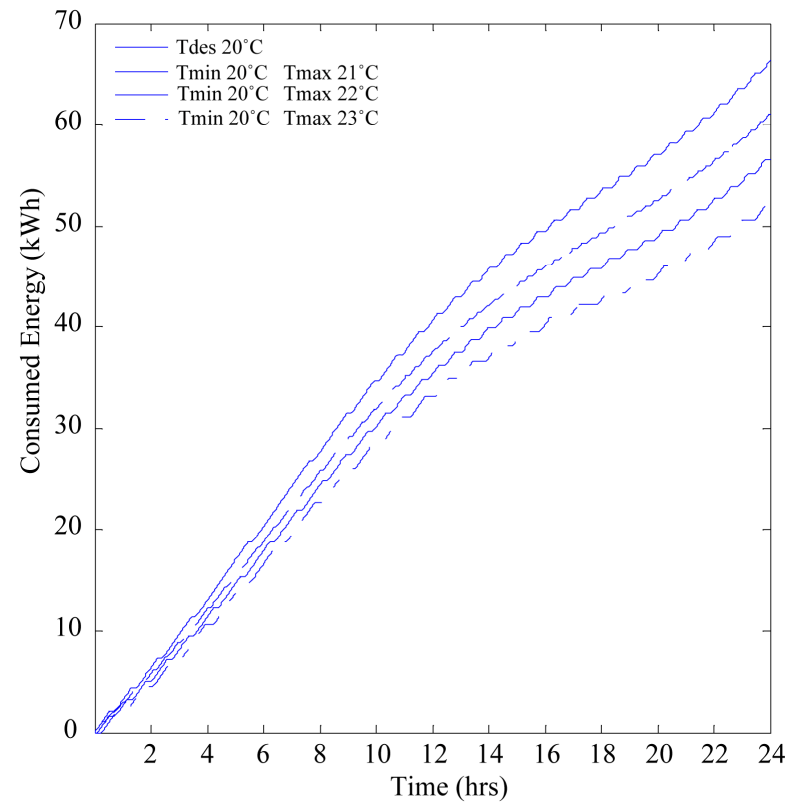

Figure 8. AC energy consumption with $\Theta \min 20^{\circ} \mathrm{C}$ and variable @max.

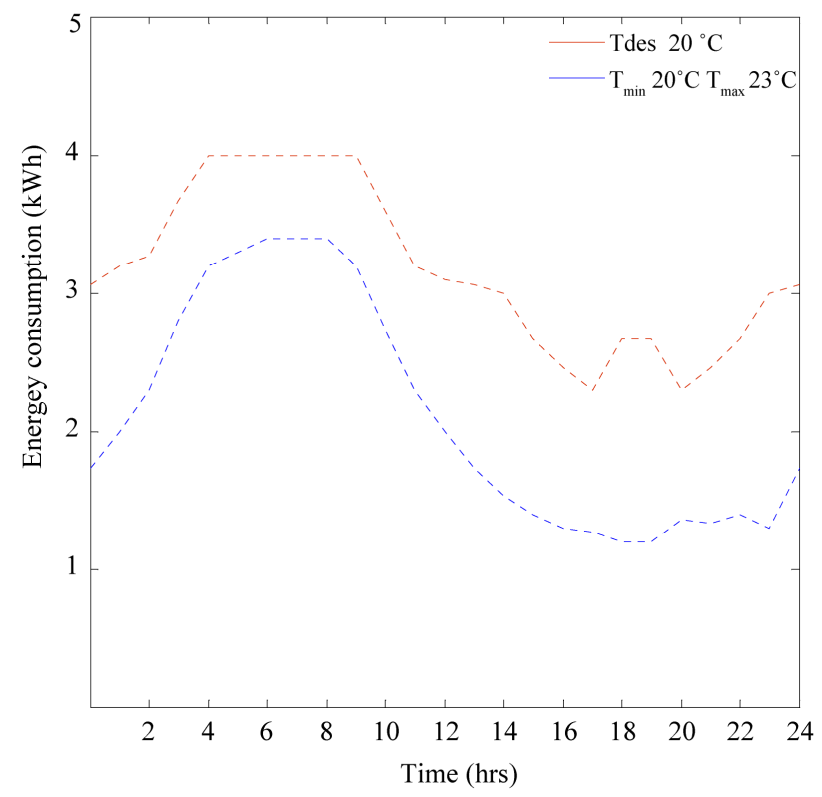

Figure 9. AC energy consumption with temperature control. 
tained. If the temperature is regulated to $\Theta_{\max } 23^{\circ} \mathrm{C}$ for 24 hours, $14 \mathrm{kWh}$ energy saving is achieved.

If excess energy consumption is detected the controller converts it to a temperature instruction to reduce the energy consumption. It acts to maintain a minimum temperature $\Theta_{\min }$ and to modify the maximum temperature $\Theta_{\max }$ within the customer thermal comfort.

During peak hours by controlling the temperature the customer consumption can be reduced.

As shown in Figure 10 the temperature control can be activated. Figure 11 shows the reduced energy consumption during the peak time.

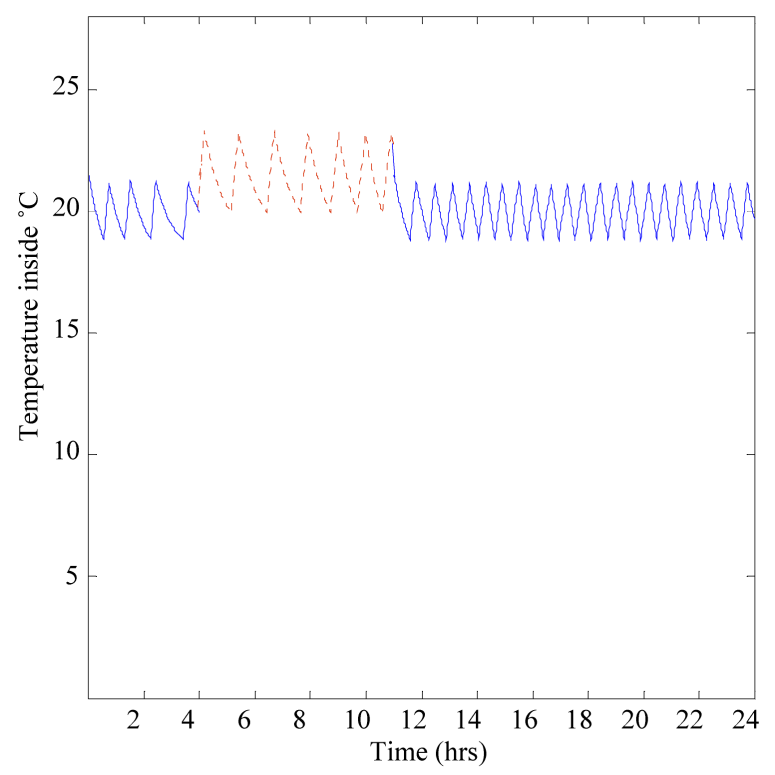

Figure 10. Temperature inside with temperature control.

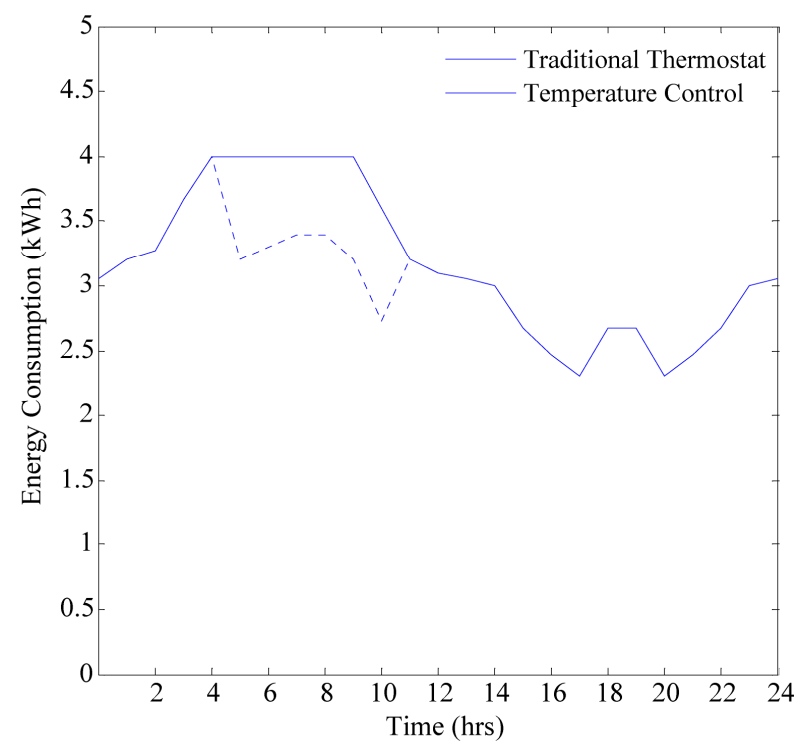

Figure 11. Reduced AC consumption during peak time.
The thermal model of a specific house data with the outside temperature captures the inside temperature, the energy usage, duty cycles and thermostat control. It can be implemented and used to find the temperature instruction to control the energy consumption.

\section{Conclusions}

This paper presents an AC energy management and control system by utilizing the web services technology. A thermal model of a house considers the house data and the outside temperature is proposed. A temperature control is presented to reduce the AC energy consumption while maintaining the customer thermal comfort. Simulation results showed significant reduction in the $\mathrm{AC}$ energy consumption while maintaining the customer thermal comfort using the proposed control method.

\section{REFERENCES}

[1] The National Energy Technology Laboratory (NETL), “A Systems View of the Modern Grid; Sensing and Measurement," March 1, 2007. http://www.netl.doe.gov/moderngrid/

[2] N. Nhat-Hai, T. Quoc-Tuan, L. Jean-Michel, V. Tan-Phu, "A Real-Time Control Using Wireless Sensor Network for Intelligent Energy Management System In Buildings," Proceedings of the IEEE Workshop on Environmental Energy and Structural Monitoring Systems (EESMS), Taranto, 9 September 2010, pp. 87-92. doi:org/10.1109/EESMS.2010.5634176

[3] K. Le, T. Tran-Quoc, J. C. Sabonnadiere, C. Kieny and N. Hadjsaid, "Peak Load Reduction by Using Air-Conditioning Regulators," Proceedings of the IEEE Mediterranean Electrotechnical Conference MELECON 2008, 5-7 May 2008, pp. 713-718.

[4] T. Tran-Quoc, J. C. Sabonnadiere, C. N. Hadjsaid and Ch. Kieny, "Air Conditioner Direct Load Control in Distribution Networks," Proceedings of the IEEE Power Tech Conference Conference 2009, Bucharest, 28 June - 7 July 2009, pp. 713-718.

[5] A. Molderink, V. Bakker, G. Maurice, M. G. C. Bosman, J. L. Hurink and G. J. M. Smit, "Management and Control of Domestic Smart Grid Technology," IEEE Transactions on Smart Grid , Vol. 1, No. 2, September 2010, pp. 109- 119. doi:org/10.1109/EESMS.2010.5634176

[6] E. Kazanavičius, A. Mikuckas, I. Mikuckienè and J. Čeponis, "The Heat Balance Model of Residential House," Information Technology and Control, Vol. 35, No. 4, 2006, pp. 391-396.

[7] M. A. A. Pedrasa, T. D. Spooner and I. F. MacGill, "Coordinated Scheduling of Residential Distributed Energy Resources to Optimize Smart Home Energy Services," IEEE Transactions on Smart Grid , Vol. 1, No. 2, September 2010, pp. 134-143. doi:org/10.1109/TSG.2010.2053053 
[8] Y. Dogan and D. Adam, "Efficient Application Integration in IP-Based Sensor Networks," Proceedings of the First ACM Workshop on Embedded Sensing System for Energy-Efficiency in Buildings (BuildSys09), Berkeley, CA, 2009.

[9] N. Priyantha, A. Kansal, M. Goraczko and F. Zhao, "Tiny Web Service: Design and Implementation of Interoperable and Evolvable Sensor Networks," Proceedingds of the 6th ACM Conference on Embedded Network Sensor Systems (SenSys08), Raleigh, NC, 2008, pp. 253-266.

[10] P. Gibbons, B. Karp, Y. Ke, S. Nath and S. Seshan, "Iris Net: An Architecture for A Worldwide Sensor Web,"
IEEE Pervasive Computing, Vol. 2, No. 4, October-December 2003, pp. 14-21.

[11] O. Asad, M. Erol-Kantarci and H. T. Mouftah, "Sensor Network Web Services for Demand-Side Energy Management Applications in the Smart Grid," Proceedings of the IEEE Consumer Communications and Networking Conference (CCNC11), Las Vegas, January 2011.

[12] "Canadian Center for Occupational Health and Safety CCOHS," August 2011.

http://www.ccohs.ca/oshanswers/phys agents/thermal co mfort.html. 